\title{
Captura de Desmodus rotundus em regiões de mata e manguezais do Estado do Maranhão: um estudo longitudinal ${ }^{1}$
}

\author{
Roberto C.N. Arruda², Thales A. Barçante ${ }^{3}$, Ana Paula Peconick ${ }^{4}$, Stela M. Pereira ${ }^{4}$, \\ José C.P. Souza ${ }^{5}$, Ticiana M. Sousa ${ }^{4}$ e Joziana M.P. Barçante ${ }^{4 *}$
}

\begin{abstract}
Arruda R.C.N., Barçante T.A., Peconick A.P., Pereira S.M., Souza J.C.P., Sousa T.M. \& Barçante J.M.P. 2013. [Desmodus rotundus capture in forest (Amazon biome) and mangrove areas in the State of Maranhão, Brazil: a longitudinal study.] Captura de Desmodus rotundus em regiões de mata e manguezais do estado do Maranhão: um estudo longitudinal. Pesquisa Veterinária Brasileira 33(5):571-574. Setor de Medicina Veterinária Preventiva, Departamento de Medicina Veterinária, Universidade Federal de Lavras, Campus Universitário, Lavras, MG 37200-000, Brazil. E-mail: joziana@dmv.ufla.br

The aim of the study was to investigate the number of captures and the control of the common vampire bat Desmodus rotundus on small farms in the municipality of Cedral, State of Maranhão, Brazil. The area was chosen because the highest number of captures in Maranhão was made there around corrals. Official control is accomplished by applying warfarin $2 \%$, a vampiricid gel, on captured bats. In this study, control records of captured hematophagous bats were analyzed, and a questionnaire was applied in the field to identify areas with the highest number of captures. From 2005 to 2010, 223 captures in 101 farms were studied; in 190 of them existed control with application of warfarin $2 \%$ gel on the bats.. There was a total of 344 attacked livestock out of 754 exposed animals, mostly cattle (49\%), followed by horses (18\%), poultry (15\%), pigs (9\%) and goats (8\%). After 6 years, the proportion of captured male and female bats was 1.08 to 1.00 , compared to 1.00 to 1.21 in the first year, what demonstrates that the treatment was more effective in females. Within three years, there was control in the number of bats in $95 \%$ of the farms, after 1 to 6 captures. The main attacked area of the animal bodies was the neck. Only $57 \%$ of the owners vaccinated their livestock. The present study concluded that (1) the control with capture of bats in corrals was effective, (2) but it is prudent to monitor the area during the first 3 months after the end of control, (3) the number of captured bats was similar to the number of recent bites, and (4) D. rotundus could attack any domestic animal, with little predilection for poultry.
\end{abstract}

INDEX TERMS: Capture, control, Desmodus rotundus, mangrove, Maranhão.

\footnotetext{
${ }^{1}$ Recebido em 27 de novembro de 2012.

Aceito para publicação em 16 de fevereiro de 2013.

${ }^{2}$ Superintendência Federal de Agricultura, Pecuária e Abastecimento no Maranhão, Praça da República 147, Bairro Diamante, São Luís, MA 65020500, Brasil. E-mail: rcnegreiros.arruda@hotmail.com

${ }^{3}$ Curso de Medicina Veterinária, Pontifícia Universidade Católica de Minas Gerais, Av. Padre Francis Cletus Cox 1661, Poços de Caldas, MG 37701355. E-mail: tbarcante@gmail.com

${ }^{4}$ Departamento de Medicina Veterinária, Universidade Federal de Lavras, Cx. Postal 3037, Lavras, MG 37200-000, Brasil. E-mails: anappeconick@ dmv.ufla.br, stelapereira@dmv.ufla.br, ticiana@dmv.ufla.br*Autor para correspondência: joziana@dmv.ufla.br

${ }^{5}$ Superintendência Federal de Agricultura, Pecuária e Abastecimento, Av. Rodrigues Alves 129, 8o andar, Rio de Janeiro, RJ 20081 250, Brasil.
}

RESUMO.- 0 presente trabalho teve por objetivos investigar o número de capturas e o controle do morcego hematófago Desmodus rotundus, em pequenas propriedades localizadas no município de Cedral no estado do Maranhão, que foi escolhido por ter sido efetuado o maior número de capturas do estado, sendo todas ao redor de currais. 0 controle oficial é realizado pela aplicação de pasta vampiricida de uso tópico a base de warfarina a $2 \%$. Para o estudo foram utilizadas fichas de controle de morcegos hematófagos capturados em currais, como também, foi aplicado um questionário a campo para identificação dos locais com maior número de capturas. No período de 2005 a 2010 foram estudados os resultados de 223 buscas ativas por $D$. 
rotundus, em 101 propriedades. Foi capturado um total de 408 morcegos hematófagos, distribuídos em 190 das 223 buscas. Em todos os espécimes de D. rotundus capturados foi realizado o tratamento, que consistiu na aplicação da pasta vampiricida, no dorso do animal. Nas propriedades estudadas, verificou-se que dos 754 animais expostos, 344 foram agredidos. Destes, os bovinos foram a maior oferta de alimento aos Desmodus (49\%), seguidos dos equídeos (18\%), aves (15\%), suínos (9\%) e caprinos (8\%). Apesar dos bovinos terem sido os mais atacados, em função do efetivo disponível, verificou-se uma preferência dos morcegos hematófagos por eqüídeos. As falhas nas capturas ocorreram onde havia poucos animais agredidos. Após análise de seis anos de realização do programa de controle com pasta vampiricida, verificou-se que a proporção de macho e fêmeas de $D$. rotundus capturados foi 1,08 machos para cada fêmea, o que demonstra que o tratamento foi mais efetivo nas fêmeas, uma vez que no primeiro ano do estudo, a proporção era de 1,21 fêmeas para cada macho. Verificou-se ainda que o controle foi realizado com sucesso em $95 \%$ das propriedades trabalhadas, com uma média de tempo de três anos de trabalho com até seis capturas por propriedade. Ao exame dos animais agredidos verificou-se que a área do pescoço dos grandes animais e dos caprinos são as mais atingidas, sugerindo o pouso direto dos morcegos sobre o animal. Uma representação pequena dos proprietários entrevistados vacinavam seus animais (57\%), e destes apenas um fazia o reforço de vacinação. Concluiu-se que nas regiões de mangue e/ou matas, o controle foi efetivo nas capturas em currais, sendo prudente verificar a ocorrência de novas agressões, dentro de 15 dias e nos primeiros três meses. No planejamento de controle de $D$. rotundus em curral, deve ser considerado que o número de morcegos capturados é semelhante ao número de mordidas recentes. Em relação às criações domésticas de subsistências, $D$. rotundus se alimenta de fontes distintas, com uma menor predileção pelas aves.

TERMOS DE INDEXAÇÃO: Capturas, controle, Desmodus rotundus, mangue, mata, reentrâncias maranhenses.

\section{INTRODUÇÃO}

Desde que Colombo descobriu a Ilha de Trinidad, em 1498, sabe-se da existência de morcegos hematófagos no hemisfério ocidental (Málaga-Alba 1954). A espécie Desmodus rotundus se distribui por toda América Latina, desde o norte do México, até o norte da Argentina (Linhart 1975, Uieda 1987). No Brasil, há relato de espoliação de morcegos hematófagos em 1543, quando da permanência de pessoas e animais em Portos dos Reis, no pantanal mato-grossense (Cabeza de Vaca 2009).

O Serviço Estadual de Saúde no Maranhão registrou que em 2005, houve 24 casos de mortes em humanos por raiva, e dentre as variantes antigênicas que são compatíveis com um painel de anticorpo monoclonal definido pelo Centro de Controle e Prevenção de Doenças (CDC). Todos os casos registrados para o Estado foram confirmados como sendo causados pela variante 3 , associada ao morcego D. rotundus. Arruda (2011) relata que a presença de D. rotundus aumenta a probabilidade em até 1,5 vezes a ocorrência da raiva em herbívoros domésticos no Maranhão.

No passado, vários métodos foram utilizados na tentativa de controle de morcegos hematófagos, como o uso de gases venenosos, explosivos, fogo, luz elétrica, querosene, vegetações espinhentas, malhas de metal ou plásticas, armas de fogos, asas de corujas e até gaviões para expulsão de morcegos da caverna (Greenhall 1970). Estes métodos foram sendo substituídos pelo envenenamento com warfarina, [3-(alfa-acetonil-benzil)-4-hidroxicumarina], em função do baixo custo da mesma. Atualmente, a warfarina tem sido utilizada na forma de gel a $2 \%$ para uso dorso lombar em bovinos e a 1\% para uso nos morcegos (Greenhall 1963, Linhart et al. 1972, Mitchell et al. 1973; Flores Crespo et al. 1974, Linhart 1975, Piccinini et al. 1985, Pompei 2009).

Hoje, no Brasil, o controle restringe-se apenas a morcegos hematófagos da espécie $D$. rotundus, por ser considerada uma espécie nociva responsável por grandes prejuízos à pecuária e à saúde pública, pela transmissão da raiva aos herbívoros e ao homem (Brasil 2009).

A pecuária no estado do Maranhão está em expansão e muitas vezes o progresso significa desmatamentos, diminuição de presas naturais, com maior exposição humana e deslocamentos de transmissores, principalmente alados para novas áreas. Neste sentido, o presente trabalho teve por objetivo investigar o número de capturas e o controle do maior transmissor da raiva em herbívoros no município de Cedral, no estado do Maranhão.

\section{MATERIAL E MÉTODOS}

Foi considerado, no presente estudo, o número total de capturas realizadas no município de Cedral constituindo-se uma amostra censo, uma vez que todas as capturas foram investigadas. As capturas são realizadas de acordo com a demanda populacional, baseada em relatos de espoliações em animais ou seres humanos no referido município, que possui uma área de $289 \mathrm{~km}^{2}$ e faz parte da Microrregião do Litoral Ocidental Maranhense, com características de mangue e floresta pré-amazônica (bioma amazônico). A população de bovídeos é constituída por cerca de 965 animais.

A coleta de dados foi realizada por meio das fichas oficiais de controle de capturas, preenchidas por técnicos da Agência Estadual de Defesa Agropecuária do Maranhão ou da Prefeitura Municipal de Cedral, no período de 2005 a 2010. Foram coletados dados referentes ao número de mordeduras recentes nos animais, às espécies de criação acometidas e aos morcegos hematófagos capturadas. A técnica de captura de morcegos hematófagos utilizada consistiu na armação de redes de neblina ao longo do curral, especificamente na parte externa, entre 1,2 a 1,5m de distância, colocadas no final da tarde. As demais espécies de quirópteros capturadas foram soltas, por volta das $24 \mathrm{~h}$, uma vez que não eram objeto de controle.

Foi aplicado um questionário a campo para identificar o tipo de área e a localização de agressões por morcegos. 0 questionário contemplou questões relacionadas ao nível de instrução, associação em sindicatos e utilização do método seletivo indireto, no controle de morcegos hematófagos (aplicação de pasta vampiricida nos animais agredidos), área do corpo agredida e tempo de retorno entre tratamento e novas espoliações. As análises, incluindo a associação entre as agressões e as espécies estudadas, foram realizadas com o auxílio do Programa GraphPad Instat $®(G S I$ 2000), 
pelo Teste Exato de Fisher, em que o nível de significância de 5\% $(\mathrm{P}<0,05)$, e o intervalo de confiança de $95 \%$.

\section{RESULTADOS}

No período de 2005 a 2010 foram realizadas 223 buscas ativas por Desmodus rotundus em 101 propriedades com problemas de mordeduras de morcegos hematófagos. Juntas, as 101 propriedades visitadas foram responsáveis por um total de 754 animais expostos, incluindo aves, bovinos, equinos, suínos e caprinos. Nenhuma das propriedades visitadas apresentou ovinos entre os animais expostos. Dos 754 animais expostos, 344 apresentaram lesão recente por $D$. rotundus. No presente trabalho verificou-se que em $67,74 \%$ dos animais agredidos, a área do pescoço dos bovinos, equinos e caprinos foram as mais atingidas. Em $32,26 \%$ as agressões foram observadas nas patas, axilas, canela e ponta de cauda.

O controle seletivo direto de $D$. rotundus aplicando uma pasta a base de warfarina $2 \%$, no dorso dos morcegos capturados, foi realizado em 190 das 223 buscas ativas. Nas outras 33 (15\%) não houve captura de morcegos hematófagos, portanto a aplicação da pasta não foi realizada. As falhas nas capturas ocorreram onde havia poucos animais agredidos.

Das 101 propriedades trabalhadas, os bovinos foram a maior oferta de alimento aos D. rotundus (49\% propriedades), seguidos dos equídeos (18\%), aves (15\%), suínos (9\%) e caprinos (8\%). Houve diferença significativa nas espoliações por $D$. rotundus entre as diferentes espécies de animais domésticos, nas propriedades estudadas (Quadro 1). Há mais bovinos a disposição dos morcegos hematófagos para serem atacados, contudo, de acordo com os resultados apresentados no Quadro 1, verifica-se uma preferência pela hemtofagia em equinos. No estudo da probabilidade de agressão entre as diferentes espécies acometidas, verificou-se que, na área estudada, as aves constituíram o grupo menos atacado. Ao se comparar a probabilidade de agressão, observou-se que os eqüídeos apresentaram uma probabilidade de serem agredidos quatro vezes maior que as aves, os bovinos e suínos três vezes e os caprinos duas vezes, indicando uma predileção dos morcegos hematófagos pelos eqüídeos.

Dentre as 190 capturas, realizadas em curral, nas quais foi realizado o controle, foi possível capturar um total de

\begin{tabular}{|c|c|c|c|}
\hline Espécies & Agredidos $\mathrm{N}^{\circ}(\%)$ & Não agredidos $\left(\mathrm{N}^{\circ}\right)$ & Total $\left(\mathrm{N}^{\circ}\right)$ \\
\hline Bovinos & $179^{\mathrm{Aa}}(48 \%)$ & 192 & 371 \\
\hline Eqüídeos & $90^{\mathrm{Bb}}(67 \%)$ & 44 & 134 \\
\hline Suínos & $31^{\mathrm{Ca}}(44 \%)$ & 39 & 70 \\
\hline Caprinos & $22^{\text {Da }}(35 \%)$ & 40 & 62 \\
\hline Aves & $18^{\mathrm{Ec}}(15 \%)$ & 99 & 117 \\
\hline TOTAL & 344 & 414 & 754 \\
\hline
\end{tabular}

As letras maiúsculas diferentes na mesma coluna (agredidos) diferem estatisticamente, quando comparado entre as espécies $(\mathrm{P}<0,05)$, e as disparidades das letras minúsculas na mesma coluna, diferem estatisticamente quando comparado por espécie em relação a agredidos e não agredidos.
408 morcegos. No ano de 2005, a proporção de fêmeas era de 1,21 para cada macho. Ao final dos seis anos de estudo, foi possível verificar que a proporção de machos e fêmeas nas capturas foi de 1,08 machos para cada fêmea. No início do controle com a pasta vampiricida, apenas quatro (29\%) dos entrevistados utilizavam essa pasta nos animais. 0 número de morcegos vampiros capturados em ação de controle no município, foi de 2,14 morcegos por captura. No estudo sobre o número de capturas e repetição do serviço por propriedade, observou-se que das 101propriedades trabalhadas, 95\% não apresentaram mais problemas com morcegos hematófagos após três anos, tendo sido realizada uma média de seis capturas por propriedade, totalizando 190 capturas. Todavia, em 39 propriedades foram realizadas somente duas repetições de capturas Embora a busca ativa com aplicação da pasta tenha sido realizada em intervalos de seis meses, para algumas propriedades, houve a necessidade de retorno com nova captura e aplicação com intervalo de até 15 dias, em função do relato de novas agressões. De todas as propriedades com relato de agressões por D. rotundus, apenas $57 \%$ dos proprietários relataram que vacinavam seus animais e destes apenas um fazia o reforço vacinal.

\section{DISCUSSÃO}

Neste trabalho, entre os anos de 2005 a 2010, 754 animais foram avaliados para a presença de lesão recente por $D$. rotundus no município de maior captura de morcegos do estado do Maranhão, Cedral. Desses animais, aproximadamente $46 \%$ apresentaram lesão, verificando-se que os bovinos foram os animais mais atacados por constituírem o grupo mais abundante. Contudo, os eqüinos, embora em menor número, foram os preferidos pelos morcegos hematófagos. Greenhal (1991) e Ueda (1992) observaram, também, predominância dos ataques a bovinos e equinos. Os morcegos escolhem e atacam geralmente aqueles animais de temperamento mais dócil e que dormem na periferia do grupo (Turner 1975).

Neste estudo observou-se que a área do pescoço dos animais foi a mais atingida, sugerindo que os morcegos pousaram direto sobre o animal. Assim, pode-se recomendar o uso de vampiricida em gel "pour-on" para auxiliar na eliminação de um maior número de $D$. rotundus possível. Em menor escala (32,26\%), verificou-se a agressão nas patas, axilas, canela e ponta de cauda, um indicativo de pouso no chão, em função do animal estar deitado. Corroborando com Pereira et al. (2010), que observaram que $85,87 \%$ dos ferimentos por $D$. rotundus ocorrem na metade superior dos bovinos.

0 número de morcegos vampiros capturados em ação de controle no município, foi de 2,14 morcegos por captura, dividindo o número de $D$. rotundus capturados (408) pelo número de mordeduras recente (304), observou-se uma média de 1,34 D. rotundus por espoliação nova, ou seja, uma média de um morcego capturado por cada mordedura fresca. Dados semelhantes foram, também, observados por Thompson et al. (1972). O controle seletivo direto de D. rotundus utilizando a pasta a base de warfarina $2 \%$ foi realizado em 190 (85\%) capturas. 
Os resultados analisados sugerem que as mortes de morcegos por envenenamento foram mais efetivas nas fêmeas. Este fato pode ser explicado pela formação de colônias agrupadas por um macho dominante. Segundo Gomes et al. (2006) as fêmeas mostraram-se mais fiéis aos abrigos e melhores disseminadoras de pasta vampiricida. Além disso, o comportamento de lamber outros indivíduos de sua espécie ocorre principalmente entre as fêmeas, garantindo a integridade do grupo e a partilha de alimento. As lambeduras estimulam o regurgitamento do alimento de uma fêmea saciada, permitindo o seu aproveitamento por outra que não tenha se alimentado. As fêmeas que não colaboram na partilha do alimento são expulsas do grupo (BRASIL, 2009). A proporção de machos e fêmeas encontrada neste trabalho foi semelhante ao observado por Gomes \& Uieda (2004).

No presente estudo, se verificou que o controle foi realizado com visitas a cada seis meses, como observado por Flores Crespo et al. (1974). Este dado difere da estratégia estabelecida pelo estado de São Paulo, que indica a captura do maior número possível de morcegos em um abrigo, e retornando ao mesmo abrigo um ano depois, onde se captura cerca de $30 \%$ de morcegos hematófagos em relação à primeira captura (Instituto Pasteur 2010). Cardoso (1995), trabalhando em praias do município de Godofredo Viana/ MA, com as mesmas características do presente trabalho, observou que em cinco meses foi possível uma redução de $70 \%$ de D. rotundus.

Em todas as propriedades visitadas foi recomendado método seletivo indireto, com a indicação do uso tópico da pasta vampiricida nos animais espoliados, bem como, uso de vacinas contra raiva. Anteriormente, apenas $29 \%$ dos proprietários utilizavam a pasta vampiricida nos animais. Como justificativa de desistência, asseguravam que os morcegos escolhiam outras áreas do corpo para agredir e/ou outros animais para realizar novas mordeduras pela vizinhança principalmente, quando a pasta era colocada em cima da ferida. Sobre o controle de D. rotundus com pasta diretamente na ferida, Piccinini et al. (1985) observaram que bovinos em cativeiro apresentam um índice de abandono, pelo morcego, de quase $26 \%$. 0 número pequeno de proprietários que vacinaram ou realizaram o reforço da vacina anti-rábica nos animais demonstra a necessidade de um maior trabalho educativo, como campanhas de vacinação contra raiva onde houver mordedura de morcegos.

Com o presente trabalho foi possível concluir que a ausência de novos ataques, caracterizada como controle efetivo nas capturas em currais, ocorre dentro de três anos após a implementação da técnica de aplicação da pasta de warfarina $2 \%$ nos morcegos hematófagos capturados. 0 sucesso do controle depende de fatores como a localização dos abrigos, dimensões e sexo dos indivíduos da colônia. É prudente que os produtores realizem o monitoramento de novos ataques em intervalos de 15 dias, nos primeiros 3 meses pós captura e, no planejamento de controle de $D$. rotundus em curral, uma vez que o número de morcegos capturados está próximo ao número de mordidas recentes nos animais, numa proporção de um macho capturado por cada fêmea.

Agradecimentos.- Ao Curso de Pós-Graduação lato sensu Especialização em Defesa Sanitária Animal - PRODESA/UFLA com apoio financeiro
MAPA-SDA/CNPq, do qual este trabalho foi fruto e à FAPEMIG-Fundação de Amaparo à Pesquisa do Estado de Minas Gerais, pelo apoio financeiro, para realização deste trabalho.

\section{REFERÊNCIAS}

Arruda R.C.N. 2011. Raiva e encefalites em herbívoros domésticos no estado do Maranhão: diagnóstico, georreferenciamento e proposição de técnica para coleta de cérebro em morcegos. Dissertação de Mestrado em Ciência Animal, Universidade Estadual do Maranhão, São Luís, MA. 71p.

Brasil 2009. Controle da Raiva de Herbívoros. Manual Técnico, Secretaria de Defesa Animal, Ministério da Agricultura, Pecuária e Abastecimento, Brasília. 124p.

Cabeza de Vaca A.N. 2009. Naufrágios e comentários (Tradução de Jurandir Soares dos Santos). 2a ed. L\&PM, Porto Alegre. 240p.

Cardoso M. 1995. Agressões humanas por morcegos hematófagos (Desmodus rotundus) no garimpo de Minas Nova e Praia do Japó, Município de Godofredo Viana, Estado do Maranhão. Universidade Estadual Paulista, Botucatu. 129p.

Flores Crespo R., Burns R.J. \& Fernández S.S. 1974. Evaluación de una técnica para combatir los vampiros en sus refugios. Boln Of. Sanit. Panam. 76(5):427-432

Gomes M.N. \& Uieda W. 2004. Abrigos diurnos, composição de colônia, dimorfismo sexual e reprodução do morcego hematófago Desmodus rotundus (E. Geoffroy) [Chiroptera, Phyllostomidae] no Estado de São Paulo, Brasil. Revta Bras. Zool. 21(3):629-638.

Gomes M.N., Uieda W. \& Latorre M.R.D.O. 2006. Influência do sexo de indivíduos da mesma colônia no controle químico das populações do morcego hematófago Desmodus rotundus (Phyllostomidae) no Estado de São Paulo. Pesq. Vet. Bras. 26(1):38-43.

Greenhall A.M. 1963. Use of mist nets and strychnine for vampire bat control in Trinidad. J. Mammal. 44(3):396-399.

Greenhall A.M. 1970. Vampire bat control: a review and proposed research program for Latin America. Proc. $4^{\text {th }}$ Vertebrate Pest Conference, West Sacramento, CA, p.41-54.

Greenhall A.M. 1991. Etologia y ecologia de los Vampiros. Anais Reunion de Consulta sobre la Atencion a Personas Expuestas a la Rabia Transmitida por Vampiros. OPS, Washinton, DC., p.9.

Linhart S.B., Flores Crespo R. \& Mitchell G.C. 1972. Control of vampire bats by topical application of an anticoagulant, chlorophacinone. Boln Of. Sanit. Panam. 6:31-38.

Linhart S.B. 1975. The biology and control of vampire bats, p.221-241. In: Baer G.M. (Ed.), The Natural History of Rabies. Vol.2. Academic Press, Georgia, USA.

Malaga-Alba A. 1954. Vampire bat as a carrier of rabies. Am. J. Public. Health 44(7):909-918.

Mitchell G.C., Burns R.J. \& Kolz A.L. 1973. Rastreo del comportamiento nocturno de los murciélagos vampiros por radiotelemetría. Tec. Pec. México 24:47.

Pereira S.N., Gitti C.B. \& Cabral M.M.O. 2010. Análise da distribuição da região dos ferimentos provocados por morcegos hematófagos Desmodus rotundus (Geoffroy, 1810) em bovinos sob condições de campo. Arqs Inst. Biológico, São Paulo, 77(2):203-208.

Piccinini R.S., Peracchi A.L., Sousa J.C.P., Albuquerque S.T., Raimundo S.D.L., Tannure A.M. \& Furtado L.L. 1985. Comportamento do morcego hematófago Desmodus rotundus (Chiroptera) relacionado à taxa de ataque a bovinos em cativeiro. Pesq. Vet. Bras. 5(4):111-116.

Pompei J.C.A. 2009. Avaliação do Vampiricida gel 1\% no controle seletivo direto de colônias de Desmodus rotundus. Dissertação de Mestrado em Ciências Veterinárias, Universidade Federal Rural do Rio de Janeiro, Seropédica, RJ. 31p.

Thompson R.D., Mitchell G.C. \& Burns R.J. 1972. Vampire bat control by systemic treatment of livestock with an anticoagulant. Science 177:806-808.

Turner D.C. 1975. The vampire bat: a field study in behaviour and ecology. Johns Hopkins University Press, Baltimore. 145p.

Uieda W. 1987. Morcegos hematófagos e a raiva dos herbívoros no Brasil. Anais do Seminário de Ciências das Faculdades Integradas de Uberaba (Fiube), Uberaba, Minas Gerais, p.13-29. 